\title{
Isolated hypopyon as an initial manifestation of relapsed acute lymphoblastic leukemia
}

\author{
Latha $S^{1}$, Karthikeyini $S^{2}$, Hercules Aro $S^{3}$, Scott J.X \\ ${ }^{1}$ Dr. Sneha Latha M, Assistant Professor, Division of Pediatric Hemato Oncology, Department of Pediatrics, \\ ${ }^{2}$ Dr. Karthikeyini Shankar, Final Year Post Graduate, Pediatrics, ${ }^{3}$ Ms. Hercules Aro S, CRRI Department of Pediatrics, \\ ${ }^{4}$ Dr. Scott Julius Xavier, Pediatrics, Fellowship in Pediatric Hemato Oncology (Australia), Professor of Pediatrics, Head, \\ Division of Pediatric Hemato Oncology; all authors are affiliated with Sri Ramachandra University.
}

Address for Correspondence: Dr. Sneha Latha M, Assistant Professor of Pediatrics, Division of Pediatric Hemato Oncoloy, Sri Ramachandra Medical Centre. E-mail id: drmslatha@yahoo.com

\begin{abstract}
The common sites of relapses of acute lymphoblastic leukemia are bone marrow, central nervous system and testis. When unusual sites like eyes are involved in extramedullary relapses, they may masquerade as hypopyon uveitis. We report a child who presented with isolated hypoyon as an initial manifestation of Acute lymphoblastic relapse, 3 months after his completion of chemotherapy. Leukemic involvement of the anterior segment of the eye should be suspected in all cases of uveitis that are refractory to treatment and particularly when a hypopyon uveitis develops in a child.
\end{abstract}

Key-words: Acute lymphoblastic leukemia, Anterior uveitis, Hypopyon, Relapse.

\section{Introduction}

With therapeutic advances, there has been considerable improvement in the survival rate of acute leukemic children [1]. This has led to an increase in variability of ocular presentations in the form of side effects of treatment and leukemic relapses being first identified as an ocular presentation [2]. Acute lympoblastic leukemias (ALL), is the most common leukemia seen in pediatric population [3]. Inspite of intensive chemotherapy, $20 \%$ of them still relapse. The relapse could be an isolated event involving the bone marrow alone or may involve central nervous system and testis. Unilateral ocular involvement as an initial manifestation of relapse in ALL is rare. We report the case of 7 year old boy who completed his chemotherapy and presented with unilateral pseudohypopyon, few months later, which was subsequently diagnosed as relapse.

\section{Case History}

A 5 year old male child presented to us with generalized lymphadenopathy and hepatomegaly in 2009. Investigations revealed leukocytosis with peripheral smear showing 60\% lymphoblasts. Bone marrow biopsy confirmed the diagnosis of Pre B CALLA positive ALL. CSF analysis was normal. Cytogenetics study revealed no abnormalities. He was treated with Children Oncology Group (COG) high risk ALL protocol. 3 months after his completion of treatment, he presented with redness, pain and photophobia in his left eye. He was seen by an ophthalmologist and was being treated symptomatically with topical eye drops and analgesics. Due to the persistence of symptoms, he was referred to a Tertiary care ophthalmology centre.

A clinical diagnosis of hypopyon was made, which aroused the suspicion of ocular relapse (fig 1). Anterior chamber fluid aspiration and cytology proved to it to be malignant cells. Bone marrow biopsy and CSF fluid analysis confirmed relapse in bone marrow and CSF. Child was started on Relapsed ALL protocol and topical steroids. With the completion of induction phase of chemotherapy, the hyopyon resolved (fig 2) and bone marrow aspiration and CSF analysis showed no blasts. Child is currently on Interim maintenance phase of chemotherapy and on regular follow up.

Manuscript received: $24^{\text {th }}$ July 2016

Reviewed: $8^{\text {th }}$ August 2016

Author Corrected; $20^{\text {th }}$ August 2016

Accepted for Publication: $1^{\text {st }}$ September 2016 


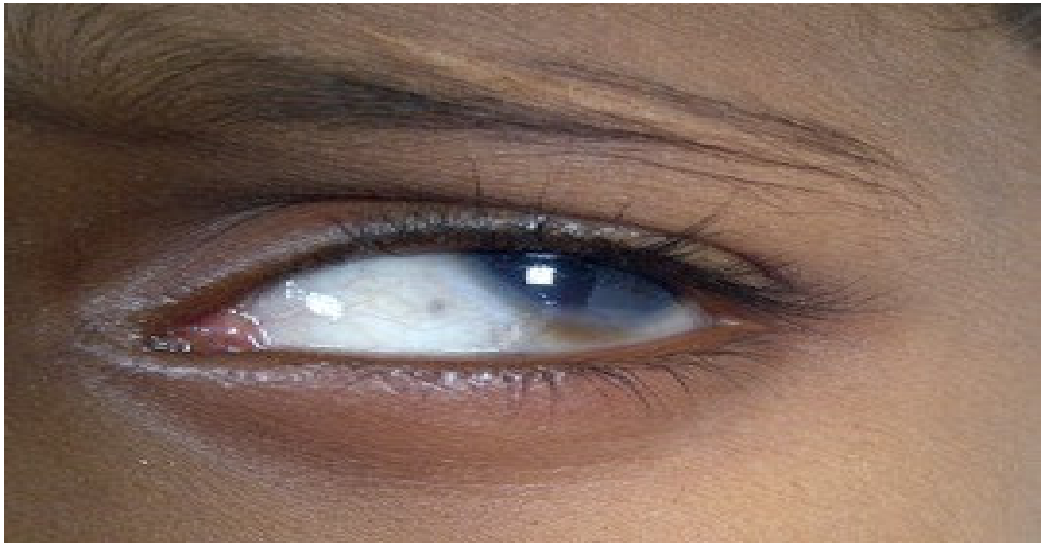

Fig-1: Hypopyon in the left eye

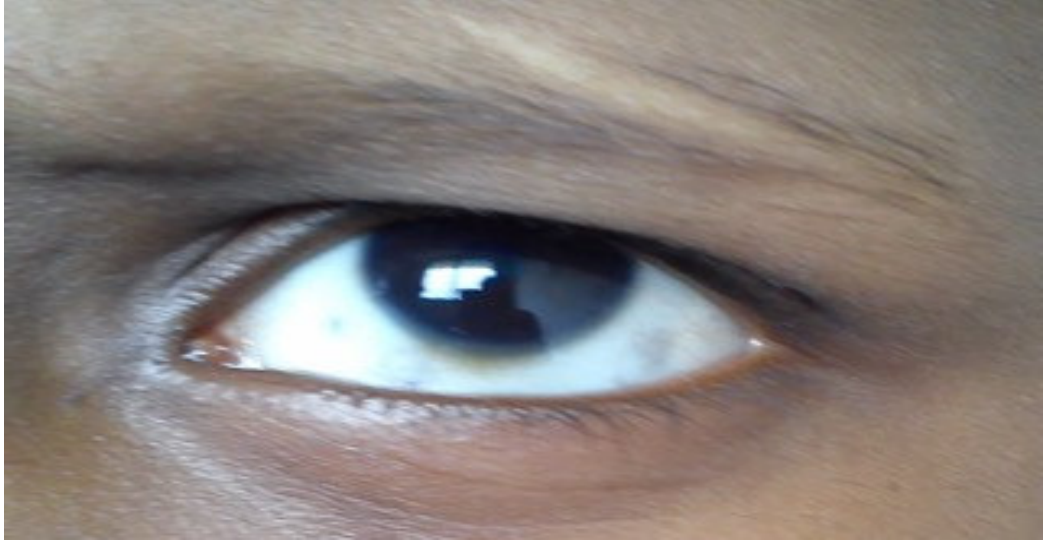

Fig-2: Resolved Hypopyon after 1 month of chemotherapy

\section{Discussion}

Eye involvement in leukemia can be of two types, primary or direct leukemic infiltration and secondary or indirect involvement. The direct leukemic infiltration can present as anterior segment uveal infiltration, oribital infiltration and neuro ophthalmic signs of CNS leukemia like optic nerve infiltration, cranial nerve palsies and papilloedema. The secondary changes are due to hematological abnormalities of leukemia such as anemia, thrombocytopenia, hyperviscosity, toxicity of various chemotherapeutic agents and immunesuppression [4]. Anterior segment is an uncommon site of extramedullary relapse , accounting for only $0.5 \%$ to $2.6 \%$ of all ALL relapses [5]. Anterior chamber involvement, particularly as in isolated finding is more common in ALL than AML. In ALL, anterior segment involvement is around $2.5 \%$ to $18 \%$ of relapsed cases, depending upon the stage of the disease [6]. The symptoms when anterior segment is involved in relapse are non specific like redness, photophobia, pain, epiphora, change in color and appearance of iris and may mimic infective or inflammatory etiology. Though the clinical findings may be varied, iritis and hypopyon are the most common.
Hypopyon is layering of white blood cells in the dependent part of the anterior chamber. It occurs in association with intraocular inflammation, infection, keratitis or malignancy. It is of value as a clinical sign because it tends to occur in association with specific conditions rather than as a general ocular inflammatory response [7]. Extramedullary relapse of acute leukaemias may masquerade as hypopyon uveitis. If hypopyon is due to ocular tumors, it consists largely of neoplastic cells, in which case it is called pseudohypopyon. A pseudohypopyon is characterized by its persistence and irregular contour, which suggests clumping of neoplastic cellular material rather than the layering of neutrophils as in anterior uveitis. The absence of posterior synechias and increased intraocular pressure helps to distinguish anterior uveitis from anterior segment leukemic infiltrates [6]. Usually clinically apparent involvement of the iris and anterior segment, particularly as an isolated finding is more common in ALL [8]. There have been reports of hypopyon uveitis as an isolated sign of relapse of AML and two reports of leukemic pseudohypopyon in CML, which may have been associated with blast crisis $[9,10]$. 
Leukaemias have been identified as the cause of uveitis in $5 \%$ of paediatric uveitis cases [11].

The high risk period for anterior chamber leukemic relapse has been found to be the first few months following completion of maintenance therapy. Eyes remain a sanctuary site for leukemic cells as they are shielded during CNS irradiation and chemotherapy agents do not penetrate the eye wall. The mechanisms by which the cells migrate into the anterior chamber are not clear. One hypothesis holds that the cells are sequestered in the long posterior ciliary vessels and pass into the anterior chamber through the iris vessels [12] Diagnosis of leukemic iris infiltration is often made clinically on slit lamp examination and should be confirmed with aqueous cytology. Leukemic involvement of the anterior segment of the eye should be suspected in all cases of uveitis that are refractory to treatment and especially when they present with hypopyon uvetis.

\section{Conclusion}

A hypopyon that fails to resolve with local or systemic corticoid steroid therapy should raise suspicion and prompt an anterior chamber tap for cytology and culture. Ocular involvement is generally associated with evidence of malignant cells at other sites and a thorough search should be made to rule out systemic and CNS involvement though the eye may be the sole site of renewed disease.

\section{Funding: Nil, Conflict of interest: Nil \\ Permission from IRB: Yes}

\section{References}

1. Hudson MM, Meyer WH, Pui CH. Progress born from a legacy of collaboration. J Clin Oncol. 2015 Aug; 23(4):2935-7. doi: 10.1200/JCO.2015.63.4535.

2. Sharma T, Grewal J, Gupta S, Murray PI. Ophthalmic manifestations of acute leukaemias: the ophthalmologist's role. Eye(Lond). 2004 Jul;18(7):66372.
3. Childhood cancer. In: Howlader N, Noone AM, Krapcho M, et al., eds.: SEER Cancer Statistics Review, 1975-2010. Bethesda, Md: National Cancer Institute,2013,Section 28.Last accessedAugust19, 2016.

4. Novakovic P, Kelly S, Taylor D. Childhood Leukaemia: relapse in the anterior segment of the eyes. Br J Opthalmol. 1989 May;73(5):354-9.

5. Ridgway EW, Jaffe N, Walton DS. Leukemic ophthalmopathy in children. Cancer. 1976 Oct;38(4): 1744-9.

6. Ramsay A, Lightman S. Hypopyon Uveitis. Surv Ophthalmol. 2001 Aug; 46(1):1-18.

7. Badeeb O, Anwar M, Farwan K, Tashkandi I, Omar A, Marzouki A. Leukemic infiltrate versus anterior uveitis. Ann Opthalmol. 1992 Aug;24(8):295-8.

8. Bunin N, Rivera G, Goode F, Hustu HO. Ocular relapse in the anterior chamber in childhood acute lymphoblastic leukemia. J ClinOncol. 1987 Feb;5(2): 299-303.

9. Ayliffe W, Foster CS, Marcoux P, Upton M, Finkelstein M, Kuperwaser M, Leqmann A. Relapsing acute myeloid leukaemia manifesting as hypopyon uveitis. Am J Ophthalmol. 1995 Mar; 119(3):361-4.

10. Lipton JH, McGowan HD, Payne DG. Ocular masquerade syndrome in lymphoid blast crisis of chronic myeloid leukemia. Leuk Lymphoma. 1995 Dec; 20(1-2):161-3.

11. Perry HD, Mallen FJ. Iris involvement in granulocytic sarcoma. Am J Opthalmol. 1979 Apr;87 (4):530-2.

12. Decker EB, Burnstine RA. Leukemic relapse presenting as acute unilateral hypopyon in acute lymphocytic leukemia. Ann Opthalmalmol. 1993 Sep; 25(9):346-9.

\section{How to cite this article?}

Latha S, Karthikeyini S, Hercules Aro S, Scott J.X. Isolated hypopyon as an initial manifestation of relapsed acute lymphoblastic leukemia. Int J Pediatr Res.2016;3(9):649-651.doi:10.17511/ijpr.2016.i09.03. 